\title{
Virtual Reality based Assembly Process Validation and Rework Assistance with consistent Data Exchange
}

\author{
Rainer Müller, Leenhard Hörauf, Attique Bashir, Martin Karkowski and Max \\ Eichenwald \\ Centre for Mechatronics and Automation gGmbH (ZeMA), Group of Assembly Systems and \\ Automation Technology, Gewerbepark Eschbergerweg 46, Geb. 9, 66121 Saarbrücken \\ m. eichenwaldezema.de
}

\begin{abstract}
Due to rising product and process complexity, assistance systems for humans are increasingly used in both the planning and execution of assembly activities or as support systems for reworking. However, developments available so far usually act as isolated solutions. Therefore a continuous dataflow between top and shop floor is not given. This article presents a comprehensive assistance system consisting of a virtual planning environment and various smart devices at an assembly workstation. In the virtual environment, the feasibility of initially created product condition graphs will be validated and a training of the repair scope to be carried out will be done. As a result, a process plan is derived from the virtual environment and transferred to an assisted workstation for reworking. The repair on the real product is carried out using the created process plan so that a continuous data flow is available. Thus the system supports the persons involved in the product analysis and work plan creation up to the execution of repair activities during rework.
\end{abstract}

Keywords: Virtual Assembly, Assembly/Disassembly Planning, Training Platform, Assistance Systems, Consistent Data Exchange

\section{Introduction}

Planning processes for assembly and disassembly are becoming more and more extensive due to increasing product complexity, variant diversity and customer-specific individualization options [1, 2]. In addition, manufacturing companies are increasingly forced to reduce development costs and time. Since assembly volumes represent an immense cost factor with regard to the product development process $[2,3]$ productivity, flexibility and efficiency must increase within the scope of the corresponding planning. However, this is becoming more and more difficult to achieve as the feasibility testing of assembly volumes also becomes more extensive. As a result, virtual development methods and tools are becoming increasingly important. The threedimensional representation within Virtual Reality (VR) enables the user to visualize and interact with the digital product in a realistic way. Time-consuming and costintensive planning with the aid of physical prototypes thus becomes superfluous. The 
possible fields of application of VR technology for manufacturing companies are very diverse. A classification according to task fields can take place according to: design (prototyping), operations management (planning, simulation, training), manufacturing processes (production flows, assembly, inspections) [4] and visualization [5].

This article focuses on the areas of visualization, training and validation of assembly scopes in a virtual environment as well as the consistent data flow to and from the VR-Application. Based on that, the use of the results for other applications can be enabled. Initially created product status graphs, in which information regarding assembly and disassembly scopes are stored, are to be validated in a virtual environment and adapted if necessary. For this purpose, the assembly scopes are created in a standardized platform and transferred to the virtual environment. To achieve a sustainable utilization of validated product condition graphs, information can be transmitted to other applications e.g. to an assisting workstation. After the initial planning phase the virtual environment can be used as a training platform for the further education of employees.

In the following, the current state of research of virtual environments in the field of assembly is described (chapter 2). Since the paper focuses the overall data flow between VR and other applications, a cognitive assistance system is described as an example system which can profit from the validated product and process scopes. An overview of the data transfer and the necessary systems is given in chapter 3 . The technological implementation of the developed VR application, functionalities and the realization of the data exchange is described in chapter 4 . A summary and outlook is given in chapter 5 .

\section{State of the art and initial situation}

This chapter highlights the current developments and areas of application in the field of virtual environments with regard to assembly planning and production. One focus of the paper is on consistent data exchange. Downstream systems should benefit from the results of the virtual environment. For this paper a cognitive assistance system in the field of rework on the shop floor was used. Therefore the last section of this chapter discusses the current state of the art of this class of assistance systems.

\subsection{Virtual Environment and Assembly Planning}

Further developments with regard to the performance of software and hardware, as well as the possibility of realistic depiction of physical products allow Virtual Environments (VE) to become present in the industrial environment. In the course of industry 4.0, VE is a fundamental technology due to the development of new potentials and its broad application spectrum. According to Nikolaos et al. [3], a technological classification of the VE can be divided into

- Virtual Manufacturing (production process),

- Virtual Prototyping (product investigations) as well as

- Virtual Assembly (assembly scope). 
The traditional procedure for planning assembly and disassembly volumes is strongly linked to the expert knowledge of the planner. A fully automatic generation, also with the help of powerful CAD programs, fails due to the mathematical formulation and utilization of this knowledge. Instead of using abstract algorithms for planning the assembly scope, Virtual Assembly (VA) supports the planner within the virtual environment. With regard to the validation of possible assembly and disassembly sequences, various VR applications can be found. One of the first systems developed is the Virtual Assembly Design Environment (VADE), in which user interaction, collision analyses and assembly tools are provided. Based on this, further VR environments exist with regard to validation, visualization and simulation of assembly scopes [6-9]. However, virtual environments are often regarded only as isolated solutions. The data transfer usually only takes place one way to the VR application, but not back again. Thus, the feedback and transfer of changes made to the assembly scope to data management systems or further applications is only insufficiently considered.

\subsection{Virtual Training System}

Products are becoming increasingly complex and thus more difficult and expensive to manufacture. The training of employees on real objects is therefore only possible to a limited extent. New technological developments for supporting training and further education are therefore becoming more important [2]. In addition to the applications for planning and development, there already exist various VR platforms for mapping training scenarios [10-13]. The digital three-dimensional model, assembly as well as disassembly sequences are simulated realistically. In addition to the purely visual representation within the VR, the increase of the user's performance and learning rate could be shown by implementing auditory and tactile feedback [7]. Assembly work requiring a high degree of psychomotor skills can be trained thoroughly (learning by doing) and cost-effectively within the VR application. The goal of virtual environments as learning platforms is always the transfer and recognition of simulated scenarios from user's in reality [2].

\subsection{Cognitive assistance systems}

In the manual assembly of complex products, the use of cognitive assistance systems (also: digital assistance systems [14]) is suitable for supporting employees. In most cases this is done by fading in assembly process information $[15,16]$. Extended systems have integrated resources for process testing and documentation, so that processes can be checked for their correct execution and logged for traceability purposes. This is intended to detect and eliminate errors on site and reduce the return rate.

The assistance systems are usually operated from the shop floor. A process list (assembly sequence) is created by the foreman or a comparable employee to enable secure assembly. Using an example of product assembly, all assembly steps are recorded step by step, together with the associated test characteristics. The next step can only be processed if the previous step with the taught-in inspection characteristics has been recognized as correct. 
Assistance systems can be used for supporting the reworking of products with many variants [17]. Nevertheless, cognitive assistance systems for the rework are so far the subject of research and hardly available on the market.

\section{Concept for the creation and validation of assembly sequences}

The main system discussed in this paper is a VR-Application for validating assembly scopes. Functionalities to visualize and edit those assembly scopes are implemented in the VR application. By using a standard exchange format, relevant data can be transferred to the VR application and validated. To achieve a flow of information from the VR to subsequent systems, the standardized graph tool $(\boldsymbol{S G T})$ serves as a platform for data exchange. In the SGT the initial lists of the assembly order and processes are created already. The SGT was previously developed at ZeMA [18]. Edited and validated assembly scopes can be reimported in the SGT and later transferred to other systems. In this case a cognitive assistance system to support employees in rework where automatically created repair plans provided to the worker allow a faster disassembly and assembly of complex, variant diverse products. A summary of those three systems is given in Fig. 1. The following chapter provides a detailed description of the three systems.

\begin{tabular}{|c|c|c|}
\hline Virtual Reality & Contmol olotfonm & Assistance-System \\
\hline $\begin{array}{l}\text { - Visualization of 3D- } \\
\text { Model } \\
\text { - Interaction with objects } \\
\text { - Possibility to edit and } \\
\text { validate assembly orders } \\
\text { - Training platform }\end{array}$ & $\begin{array}{l}\text { - Data hub } \\
\text { - Graphical interface for } \\
\text { visualization of product, } \\
\text { process, resource } \\
\text { - Data import and export } \\
\text { via JSON }\end{array}$ & $\begin{array}{l}\text { - Information about } \\
\text { processes } \\
\text { - Event driven resource } \\
\text { parametrization } \\
\text { - Process checking } \\
\text { - Documentation }\end{array}$ \\
\hline 七 & & \\
\hline
\end{tabular}

Fig. 1 Central characteristics of the virtual environment, standardized graph tool (SGT) and assistance system

\section{Implementation of the environment for the creation and validation of the assembly sequences and transfer of the work plan into reworking}

The following section gives an overview over the previously described systems. Furthermore, the continuous data exchange between the three subsystems is described. 


\subsection{Scope of functions within the Virtual Environment}

The virtual environment offers the possibility of visualization as well as the validation and editing of the assembly scope. The displayed product can be assembled and disassembled step by step within VR-Application according to the planning data created in the SGT tool. As shown in Fig. 2(a) the main functionalities are "Move all" (scaling and translation of the object in space), "Teleport" (jumping movement of the user, see Fig. 2(b)), as well as "Assembly" (evaluation assembly sequence) and "Reset". Color coding of successive components or assemblies makes visualization easier. Relevant process steps are displayed in a toolbar (see Fig. 2(c)). The correctness of these can thus be continuously validated. If it is necessary to edit them, another process can be selected and saved. Furthermore, there is the option to adjust the given assembly sequence. Based on the current product status, a subsequently planned component or assembly can either be acknowledged or, if desired, replaced by an object determined by the user. For this purpose, all of the following components are displayed transparently, a chosen component for replacement is highlighted (see Fig. 2(d)). Because of this functionality the user is able to step through the parts, so that the current part will be colored and selectable. If the assembly scopes are valid, they can be confirmed and saved by the planner. Verified assembly sequences are saved in tabular form. This makes it possible to identify corresponding product status paths within the JSON file and to mark these with corresponding information regarding there validity. The marking is done by coloring of the respective edges/processes (see Fig. 3(b)).

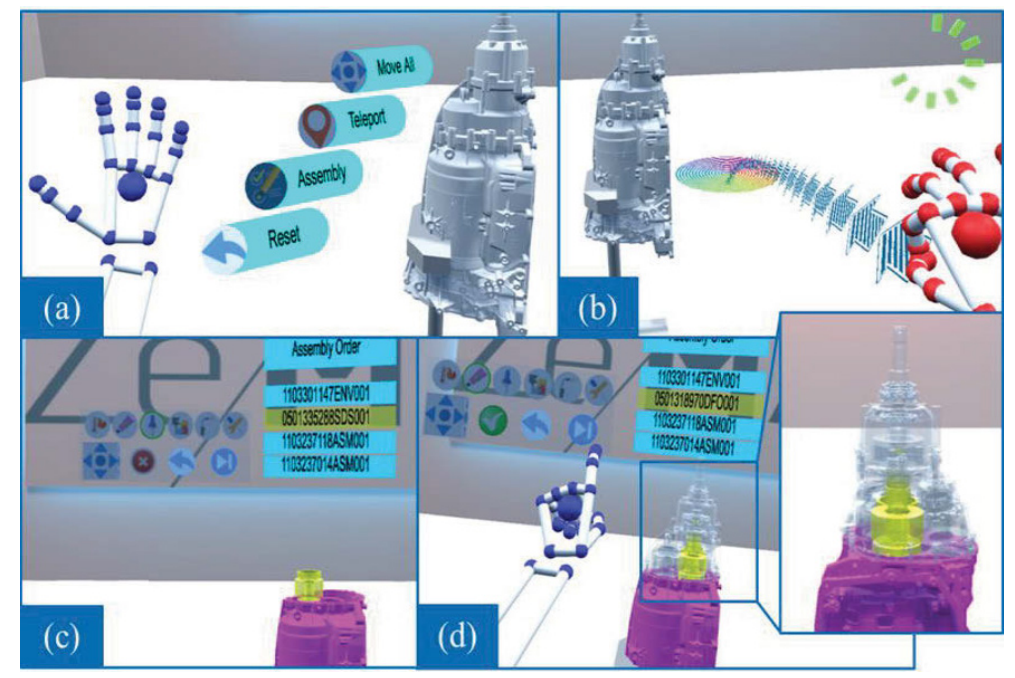

Fig. 2 Functionalities of the Virtual Environment - (a) Menu, (b) Teleport, (c) Visualization of assembly scopes, (d) Editing process

Furthermore, validated assembly scopes can be used as training scenarios. Due to higher product complexities and more variants, employees need extensive training. An alternative to training on real prototypes can be the virtual environment. 
The setup for the virtual environment includes VR glasses, two sensors to detect the position of the user, as well as a Leap Motion sensor for the detection of hand movements and gestures. On one hand, this enables intuitive handling of the application, and on the other hand, it allows to assign specific functionalities in different gestures. The engine is implemented in the Unity development environment.

\subsection{Development and setup of a planning tool for the creation of graph-based assembly sequences}

The standardized graph tool is used to model and depict the relationships between the product, the required processes and the required operating resources (see Fig. 3(a)). To model and edit the assembly sequences, the user can select and insert elements from a configurable selection of product, process and resource templates. In addition to the visual adjustments of the inserted elements, the user can dynamically assign attributes to the elements. Such attributes can contain product-specific information such as product id, included parts of the assembly, manufacturer, etc. Different edges can be used to model the relationships (processes) between the product states. The name of a specific process which is used to get from product state $\mathrm{n}$ to product state $\mathrm{n}+1$ can be entered here. To simplify the representation and structuring of elements, it is possible to hide different elements and to nest or cluster them hierarchically via father-child relationships. Created graph structures can be both exported and imported as JSON (JavaScript Object Notation) objects. With implementing the planning tool as a Web application, it can be used on a variety of different platforms.

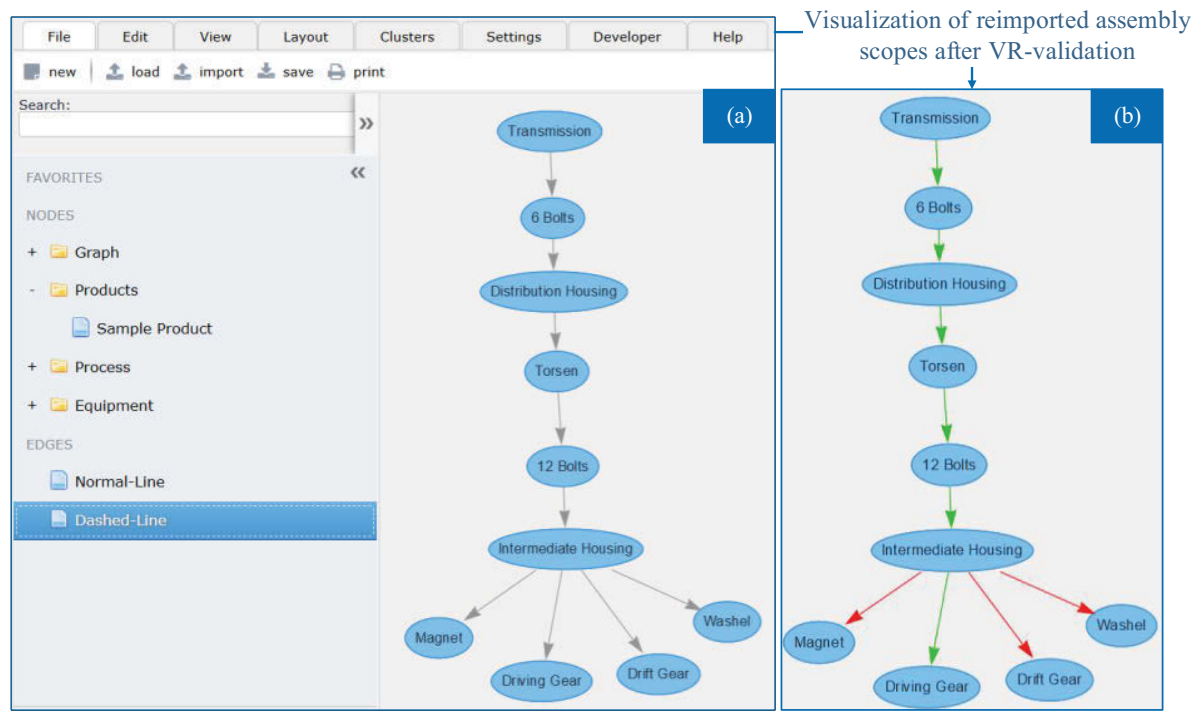

Fig. 3 Interface of standardized graph tool (SGT), (a) Product states of transmission, (b) valid (green) and non-valid (red) assembly scopes based on VR-Validation 


\subsection{Data Transfer between Virtual Environment and SGT-Platform}

Using the virtual environment requires two input formats. A three-dimensional model of the product to be viewed (.obj file), as well as the product states defined in the beginning within the SGT platform. The associated assembly volumes are exported from the SGT platform on the basis of the standardized exchange format JSON.

The different possibilities of (dis-)assemble a product result in a branched representation of product states within the SGT platform. In order to visualize all of the reasonable defined scopes in the SGT platform, each path is exported as a linear lists. Therefore the exported JSON file gets parsed and transferred in multiple .csv files, related to the number of possible paths. Those .csv-files are loaded one after another into the virtual environment. Validating of editing the assembly scopes means, that relevant information about assembly order and process will be assigned to the corresponding .csv file. After all of the lists are checked, an algorithm will loop over all of the .csv files an extract the changes. Therefore the changes can also applied to the JSON file. Valid lists are highlighted by a green color, invalid lists are highlighted red. Reimporting the adapted JSON file in the SGT gives the opportunity to quickly understand/visualize which product state branches are valid and which are not (see Fig. 3(b)). An overview of the data exchange between the systems is shown in Fig. 4.

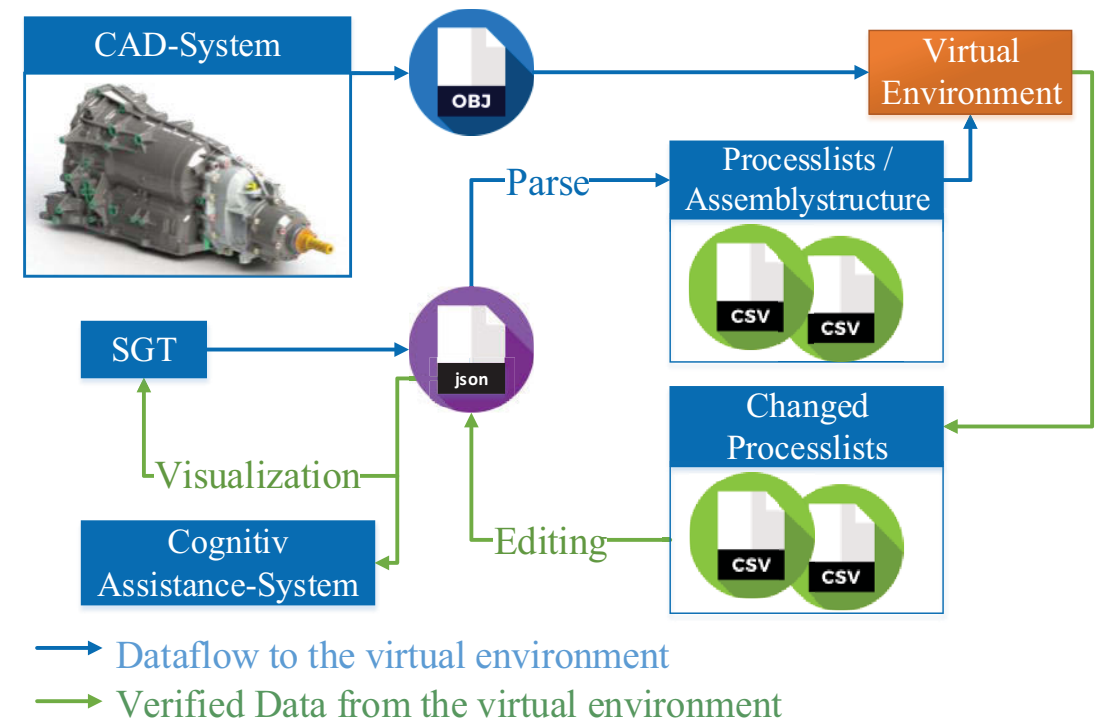

Fig. 4 Information flow between virtual environment, SGT and subsequent systems (e.g. an assistance system)

\subsection{Transferring the routing to rework}

The edited JSON-file enables the use of validated assembly scopes for further applications, e.g. the cognitive assistance system for rework. For this assistance system various product states that the employee can reach during the repair of a product must 
take into account. Therefore, a product structure is used instead of a classical assembly priority graph. The product structure is composed of nodes and edges, with the nodes representing the respective product status and the edges representing the assembly processes leading to a status change. In the first step, the employee enters an error code into the assistance system via the Co-worker guidance system (see Fig. 5) The required repair plan is then output via a database query. This controls the necessary resources in digital form and provides the employee with the necessary process information via the Co-worker guidance system.

In the technical implementation, the graph is stored in a graph and document database. The objects are organized in four categories: inform (informing the employee), parametrize (parameterizing equipment), validate (process testing) and document (documenting processes), since these four areas are covered by the assistance system. Each of these objects has a sub-object "address" and "parameter", which contain the resource to be controlled as well as the necessary parameters. The graph database allows for queries containing graph operations. For example, the shortest path from a product state to the target state can be output with a query.

The validated work plan from the virtual environment is searched by a JSON parser, whereby the nodes are written to the database and the processes are appended to the edges. The graph structure is set up with the available data and made available to the control program for operative operation.

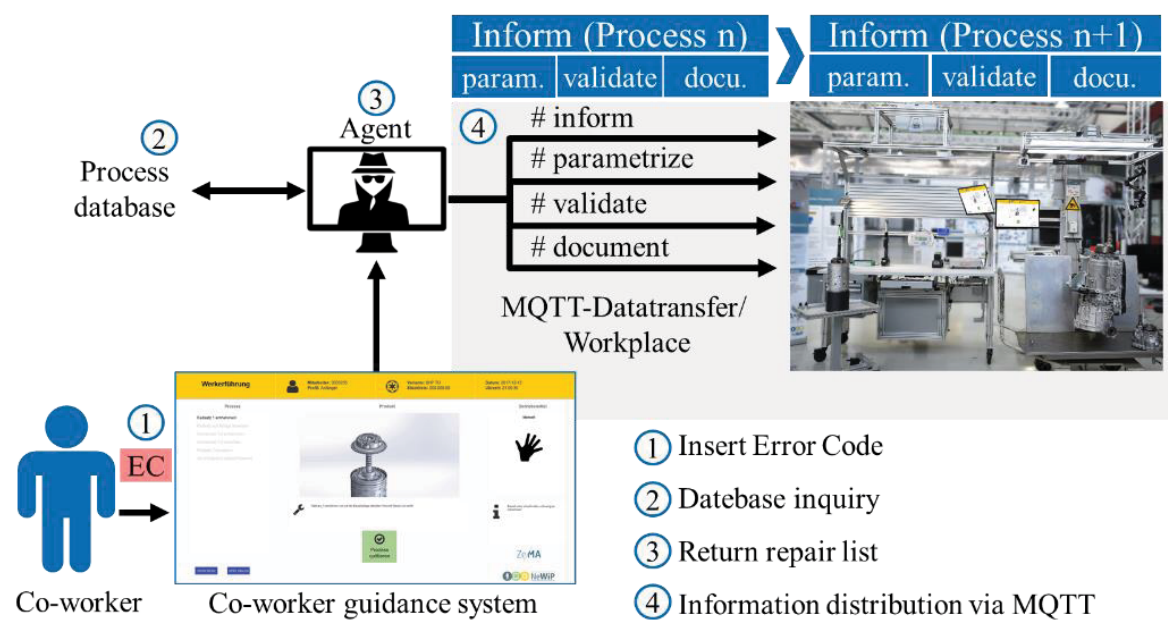

Fig. 5 Architecture of the cognitive coworker assistance system

\section{Conclusion and Outlook}

In this article, a VR-supported procedure for the feasibility assurance of reworking processes by consistent data exchange was described. The central element of the planning was a graph tool that allows the representation of product and process relationships and the input of their relevant data. The process relationships are stored as a graph together with the corresponding data in a JSON formatted text file. This serves 
as an exchange file for other systems. It is loaded into the VR environment, where a planner can start simulating the (dis-)assembly processes. Step by step, individual processes are validated and returned to the graph-based planning system as feasible or non-feasible. In addition, the virtual environment can be used as a training platform. Employees can train on already validated assembly scopes to achieve a faster understanding of the complexity of the product.

An updated graph can be transferred to other systems. This is shown on the example of a rework system, whereby data is transferred for assistance (information and process testing) as well as for process execution and documentation.

In the future, data from the rework area should be able to be fed back. This would allow optimization potentials, uncovered in rework, to be reported in a standardized way and introduced transparently in rework. Furthermore, a process list generation has to be optimized so that the minimum number of process lists is generated, in order to make the validation process effortless and repeatable.

\section{Acknowledgment}

This paper was written in the framework of the research project KomZetSaar (Funding code 01MF117003A), which is funded by the Federal Ministry of Economic Affairs and Energy (BMWi) and supervised by the lead partner German Aerospace Center (DLR).

\section{References}

1. Rademacher, M.H.: Virtual Reality in der Produktentwicklung. Instrumentarium zur Bewertung der Einsatzmöglichkeiten am Beispiel der Automobilindustrie. Springer Fachmedien Wiesbaden, Wiesbaden (2014)

2. Thomas, O., Metzger, D., Niegemann, H. (eds.): Digitalisierung in der Aus- und Weiterbildung. Springer Berlin Heidelberg, Berlin, Heidelberg (2018)

3. Bilalis Nikolaos: Industrial applications' simulation technologies in virtual environments. Part II: Virtual Manufacturing and Virtual Assembly (2003)

4. Mujber, T.S., Szecsi, T., Hashmi, M.s.J: Virtual reality applications in manufacturing process simulation. Journal of materials processing technology, 1834-1838 (2004)

5. Ovtcharova, J.G.: Virtual Engineering: Principles, Methods and Applications. DS 60: Proceedings of DESIGN 2010, the 11th International Design Conference (2010)

6. Sankar J., Yong W., Uma J.: VADE: A Virtual Assembly Design Environment. IEEE computer graphics and applications, 44-50 (1999)

7. Al-Ahmari, A.M., Abidi, M.H., Ahmad, A., Darmoul, S.: Development of a virtual manufacturing assembly simulation system. Advances in Mechanical Engineering (2016). https://doi.org/10.1177/1687814016639824

8. Jayasekera, R.D.M.D., Xu, X.: Assembly validation in virtual reality-a demonstrative case. International Journal of Advanced Manufacturing Technology (2019). https://doi.org/10.1007/s00170-019-03795-y

9. Sá, A.G., Zachmann G.: Virtual Reality as a Tool for Verification of Assembly and Maintenance Processe. Computers \& Graphics 23, 389-403 (1999)

10. Abidi, M.H., Al-Ahmari, A., Ahmad, A., Ameen, W., Alkhalefah, H.: Assessment of virtual reality-based manufacturing assembly training system. International Journal of Advanced Manufacturing Technology (2019). https://doi.org/10.1007/s00170-019-03801-3 
11. Im, T., An, D., Kwon, O-Y., Kim, S.-Y. (eds.): A Virtual Reality based Engine Training System. A Prototype Development \& Evaluation. 9th International Conference on Computer Supported Education, Porto, Portugal, 21.04.2017 - 23.04.2017. SCITEPRESS - Science and Technology Publications (2017 - 2017)

12. Brough, J.E., Schwartz, M., Gupta, S.K., Anand, D.K., Kavetsky, R., Pettersen, R.: Towards the development of a virtual environment-based training system for mechanical assembly operations. Virtual Reality (2007). https://doi.org/10.1007/s10055-007-0076-4

13. Li, Z., Wang, J., Yan, Z., Wang, X., Anwar, M.S.: An Interactive Virtual Training System for Assembly and Disassembly Based on Precedence Constraints. In: Gavrilova, M., Chang, J., Thalmann, N.M., Hitzer, E., Ishikawa, H. (eds.) Advances in Computer Graphics, vol. 11542. Lecture Notes in Computer Science, pp. 81-93. Springer International Publishing, Cham (2019)

14. Apt, W., Bovenschulte, M., Priesack, K., Weiß, C., Hartmann. E.A.: Einsatz von digitalen Assistenzsystemen im Betrieb. Im Auftrag des Bundesministeriums für Arbeit und Soziales. Forschungsbericht 502 (2018)

15. Müller, R., Vette-Steinkamp, M., Hörauf, L., Speicher, C., Bashir, A.: Intelligent and Flexible Worker Assistance Systems - Assembly Assistance Platform for Planning Assisted Assembly and Rework as Well as Execution of a Worker-Centered Assistance. In: Proceedings of the 13th International Joint Conference on Computer Vision, Imaging and Computer Graphics Theory and Applications. International Conference on Human Computer Interaction Theory and Applications, Funchal, Madeira, Portugal, 27.01.2018 29.01.2018, pp. 77-85. SCITEPRESS - Science and Technology Publications (2018 2018). https://doi.org/10.5220/0006613900770085

16. Bertram, P., Birtel, M., Quint, F., Ruskowski, M.: Intelligent Manual Working Station through Assistive Systems. IFAC - PapersOnLine (2018). https://doi.org/10.1016/j.ifacol.2018.08.253

17. Müller, R., Vette-Steinkamp, M., Hörauf, L., Speicher, C., Bashir, A.: Worker centered cognitive assistance for dynamically created repairing jobs in rework area, vol. 72, pp. 141-146, Procedia CIRP (2018). https://doi.org/10.1016/j.procir.2018.03.137

18. Müller, R., Hörauf, L., Speicher, C., Obele, J.: Communication and Knowledge Management Platform for Concurrent Product and Assembly System Development, vol. 28, pp. 107-113, Procedia Manufacturing (2019).https://doi.org/10.1016/j.promfg.2018.12.018

Open Access This chapter is licensed under the terms of the Creative Commons Attribution 4.0 International License (http://creativecommons.org/licenses/by/4.0/), which permits use, sharing, adaptation, distribution and reproduction in any medium or format, as long as you give appropriate credit to the original author(s) and the source, provide a link to the Creative Commons license and indicate if changes were made.

The images or other third party material in this chapter are included in the chapter's Creative Commons license, unless indicated otherwise in a credit line to the material. If material is not included in the chapter's Creative Commons license and your intended use is not permitted by statutory regulation or exceeds the permitted use, you will need to obtain permission directly from the copyright holder.

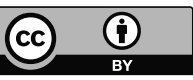

